\title{
Aberrant Expression of Trefoil Factor Family 1 in Biliary Epithelium in Hepatolithiasis and Cholangiocarcinoma
}

\author{
Motoko Sasaki, Koichi Tsuneyama, and Yasuni Nakanuma \\ Department of Human Pathology, Kanazawa University Graduate School of Medicine, Kanazawa, Japan
}

\begin{abstract}
SUMMARY: Stepwise progression of intrahepatic cholangiocarcinoma (ICC) has been proposed in hepatolithiasis. We examined the participation of trefoil factor family 1 (TFF1), which is critical for mucosal protection and tumor suppression in the stomach, in the development and progression of ICC. We used 16 livers of ICC with hepatolithiasis, 11 of biliary epithelial dysplasia with hepatolithiasis, 16 of hepatolithiasis without dysplasia or carcinoma, 18 of ICC without hepatolithiasis, and 39 control livers. TFF1 expression in the biliary epithelium was increased in hepatolithiasis compared with control livers $(p<0.01)$. In biliary epithelial dysplasia and noninvasive ICC with hepatolithiasis, TFF1 was extensively expressed and MUC5AC gastric mucin was usually colocalized with TFF1. However, TFF1 expression was significantly decreased in invasive ICC despite preserved expression of MUC5AC. A total of four missense mutations were detected: three in two noninvasive ICC with hepatolithiasis (28.6\%) and one in invasive ICC (11\%). Loss of heterozygosity of the TFF1 gene was not detectable. The decreased expression of TFF1 in invasive ICC may be explained by the methylation of the TFF1 promoter region. Up-regulation of TFF1 coupled with MUC5AC in biliary epithelium in hepatolithiasis, biliary epithelial dysplasia, and noninvasive ICC may reflect the gastric metaplasia and early neoplastic lesion. Under such conditions, decreased TFF1 expression may lead to increased cell proliferation and then to the invasive character of ICC. (Lab Invest 2003, 83:1403-1413).
\end{abstract}

$T$ here is increasing evidence that trefoil factor family (TFF) peptides are important in the mucosal defense and repair of the gastrointestinal tract (Podolsky 1999; Poulsom et al, 1996; Wright et al, 1997). TFF peptides are small and stable molecules that have one or two trefoil motifs with six cysteine residues forming three disulfide bonds and a characteristic three-loop structure (Thim, 1989). These peptides are synthesized in mucus-secreting cells and secreted onto the epithelial surface together with the mucus (Podolsky, 1999; Poulsom et al, 1996). In humans, three trefoil peptides have been identified: TFF1 (originally called pS2); TFF2 (formerly spasmolytic peptide); and TFF3 (previously called intestinal trefoil factor) (Poulsom and Wright, 1993; Wright et al, 1997). All of the three genes encoding human trefoil peptides are clustered within a region of $55 \mathrm{~kb}$ on chromosome 21q22.3 (Beck et al, 1996; Gott et al, 1996; Seib et al, 1997).

TFF1, which is mainly produced in the gastric surface epithelium, is up-regulated at sites of injury, such as peptic ulcers (Podolsky, 1999; Poulsom et al, 1996; Rio et al, 1991; Wright et al, 1993, 1997), and has a protective effect against gastric mucosal damage (Babyatsky et al, 1996). In addition, TFF1 is suggested

DOI: 10.1097/01.LAB.0000092230.59485.9E

Received November 1, 2002

Address reprint requests to: Dr. Y. Nakanuma, Department of Human Pathology, Kanazawa University Graduate School of Medicine, Kanazawa 920-8640, Japan.E-mail: pbcpsc@kenroku.kanazawa-u.ac.jp to have tumor-suppressor properties. In fact, TFF1 knockout mice developed multiple gastric adenomas and carcinomas (Lefebvre et al, 1996). The region 21q22, in which the TFF1 gene is located, is commonly deleted in human gastric cancer (Nishizuka et al, 1998; Sakata et al, 1997). Recently, Park et al (2000) reported frequent somatic mutations confined to the loop I and loop II structure of TFF1 and loss of heterozygosity $(\mathrm{LOH})$ at the TFF1 gene, and suggested that genetic alteration of TFF1 may lead to gastric mucosal barrier defects and contribute to the pathogenesis of gastric cancer. In fact, TFF1 suppresses the growth of a human gastric cancer cell line (Calman et al, 1999; Taupin et al, 2001). Furthermore, recent reports suggest that methylation of the TFF1 promoter region may underlie the loss of TFF1 (Carvalho et al, 2002; Fujimoto et al, 2000).

In hepatolithiasis, which is not uncommon in the Far East (Nakanuma et al, 1994, 1988; Nakayama et al, 1986), intrahepatic cholangiocarcinoma (ICC) is known to develop in approximately $10 \%$ of patients (Nakanuma et al, 1985; Terada and Nakanuma, 1992). Biliary epithelial dysplasia is being accepted as a precursor lesion of ICC in hepatolithiasis (Nakanuma et al, 1985, 1994; Terada and Nakanuma, 1992). Stepwise development and progression through biliary epithelial dysplasia, noninvasive ICC, and invasive ICC has been proposed in hepatolithiasis. Increased cell kinetics and altered expression of oncofetal markers (Nakanuma et al, 1985; Terada and Nakanuma, 1992) and MUC5AC gastric-type mucin gene (Sasaki et al, 1998) were reported, but the key event and exact 
mechanism during this carcinogenesis remain obscure. Although there have been a few studies regarding TFF1 expression in the biliary tract (Seitz et al, 1991; Srivatsa et al, 2002), no studies have been reported on the pathologic roles of TFF1 in the development of ICC arising in chronic biliary diseases.

In this study, we evaluated the significance of TFF1 in the development of ICC in hepatolithiasis by examining the expression of TFF1 protein and mRNA. A mutational analysis, loss of $\mathrm{LOH}$ analysis, and promoter methylation analysis for the TFF1 gene were also performed.

\section{Results}

\section{Expression of TFF1 at the Protein and mRNA Levels}

Expression of TFF1 at the Protein Level. Table 1 summarizes the incidence and the extent of TFF1 expression.

Control livers: In normal livers, most biliary epithelial cells (BEC) did not express TFF1, except for a few BEC in $5(18.5 \%)$ of 27 livers. TFF1 was expressed faintly in the supranuclear cytoplasm of the BEC (Fig. 1A) and also focally in peribiliary glandular cells in normal livers. BEC of the small bile ducts did not express TFF1 (Fig. 1B). Almost none of the BEC in the large and small bile ducts in livers with extrahepatic biliary obstruction expressed TFF1. Livers with hepatocellular carcinoma failed to express TFF1 at all.

Hepatolithiasis: BEC in the large bile ducts expressed TFF1 in all of the hepatolithiasis cases, and the extent was significantly higher compared with those in control livers $(p<0.01)$. TFF1 was expressed clearly in the supranuclear cytoplasm as fine vesicles and also at the apical region of BEC in the large bile ducts and was similarly expressed in peribiliary glands, focally (Fig. 2A). At the margin of mucosal erosion of the large bile ducts in hepatolithiasis, TFF1 was expressed markedly (Fig. 2B). BEC in stone- containing bile ducts also showed stronger expression of TFF1.

Biliary epithelial dysplasia in hepatolithiasis: TFF1 was extensively expressed in biliary epithelial dysplasia in all cases (Fig. 3). Most of the dysplastic cells expressed TFF1 strongly. TFF1 was expressed as fine cytoplasmic vesicles, and the expression was accentuated in the supranuclear region of dysplastic cells (Fig. 3). The extent of TFF1 expression was significantly higher when compared with that of BEC in the large bile ducts in hepatolithiasis and control livers $(p<0.01)$.

Noninvasive ICC with hepatolithiasis: All noninvasive ICC expressed TFF1 extensively, and the extent of TFF1 expression was significantly higher when compared with invasive ICC and BECs in hepatolithiasis and control livers $(p<0.01)$. Augmented TFF1 expression was seen as fine vesicles in the supranuclear cytoplasm and was focally accentuated the apical region of carcinoma cells (Fig. 4A).

Invasive ICC with hepatolithiasis: Six (60\%) of 10 invasive ICC with hepatolithiasis expressed TFF1, but the extent of expression was significantly decreased compared with noninvasive ICC and dysplasia $(p<$ 0.01). In invasive ICC, most invasive carcinoma cells did not express TFF1, whereas carcinoma cells of the noninvasive area expressed TFF1 extensively (Fig. 4C). Among the invasive ICC with hepatolithiasis, TFF1 expression was decreased in poorly differentiated ICC compared with well-differentiated to moderately differentiated ICC. TFF1 expression was seen as fine cytoplasmic vesicles in the supranuclear area and was focally accentuated at the apical region of carcinoma cells, when present.

Invasive ICC alone (without hepatolithiasis): TFF1 was expressed in $66.7 \%$ of invasive ICC alone, and the extent was almost similar to that of invasive ICC with hepatolithiasis. There was no significant difference in TFF1 expression between ICC of hilar type and

Table 1. Expression of TFF1 in ICC and Biliary Epithelial Dysplasia Associated with Hepatolithiasis, ICC Alone, BEC in Hepatolithiasis and Control Livers

\begin{tabular}{|c|c|c|c|c|c|c|}
\hline & \multirow[b]{2}{*}{ (n) } & \multirow{2}{*}{$\begin{array}{l}\text { Positive rates } \\
\text { (\%) }\end{array}$} & \multicolumn{4}{|c|}{ Extent of expression } \\
\hline & & & 0 & $1+$ & $2+$ & $3+$ \\
\hline \multicolumn{7}{|l|}{ BEC in large bile ducts } \\
\hline Normal livers & 27 & $18.5 \%$ & 22 & 5 & 0 & 0 \\
\hline EBO livers & 12 & $8.3 \%$ & 11 & 1 & 0 & 0 \\
\hline Hepatolithiasis (nondysplastic) ${ }^{a}$ & 16 & $100 \%$ & 0 & 13 & 3 & 0 \\
\hline $\begin{array}{l}\text { Biliary epithelial dysplasia in } \\
\text { hepatolithiasis }{ }^{b}\end{array}$ & 11 & $100 \%$ & 0 & 1 & 2 & 8 \\
\hline \multicolumn{7}{|l|}{ ICC with hepatolithiasis } \\
\hline Noninvasive ICC ${ }^{b}$ & 6 & $100 \%$ & 0 & 0 & 5 & 1 \\
\hline Invasive ICC $^{C}$ & 10 & $60 \%$ & 4 & 5 & 1 & 0 \\
\hline Invasive ICC alone ${ }^{C}$ & 18 & $66.7 \%$ & 6 & 7 & 4 & 1 \\
\hline Hepatocellular carcinoma & 11 & 0 & 11 & 0 & 0 & 0 \\
\hline
\end{tabular}

The rate of TFF1-expressing cells is $0,<5 \% ; 1+, 5-30 \% ; 2+, 30-70 \% ; 3+,>70 \%$; ICC, intrahepatic cholangiocarcinoma; EBO, extrahepatic biliary obstruction.

${ }^{a} p<0.01$ vs biliary epithelial cells in control livers.

${ }^{b} p<0.01$ vs biliary epithelial cells in control livers and hepatolithiasis.

${ }^{c} p<0.01$ vs biliary epithelial dysplasia and noninvasive CC. 

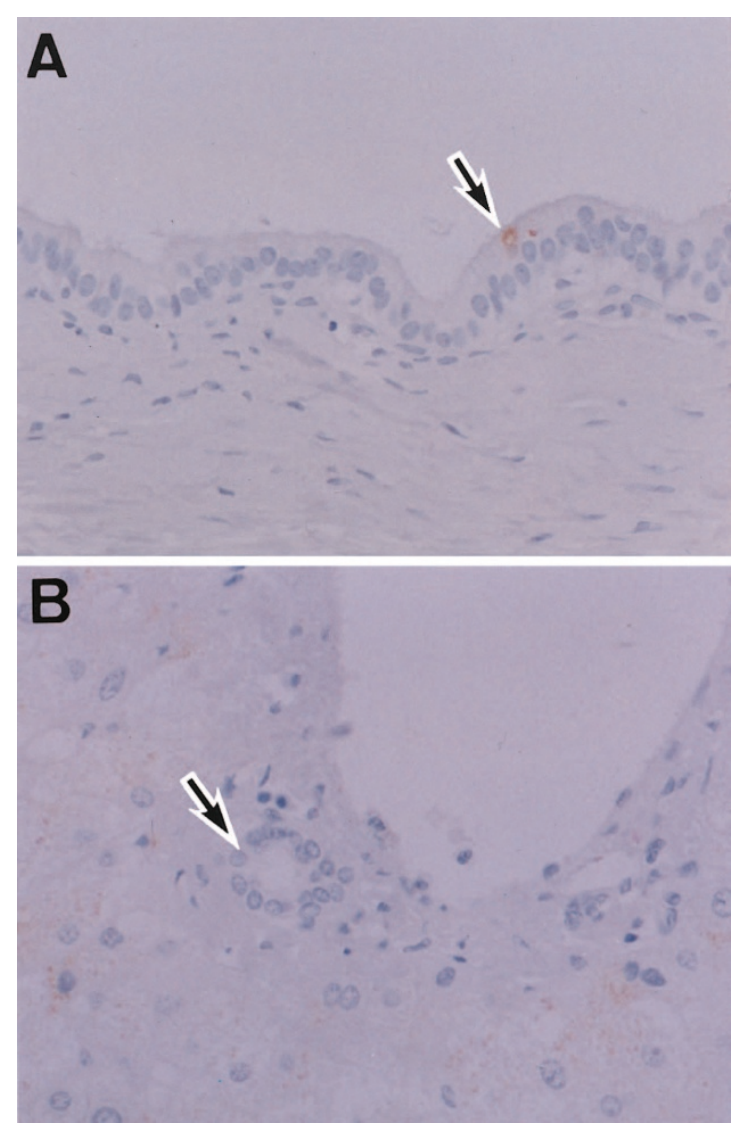

\section{Figure 1}

Trefoil factor family 1 (TFF1) expression in control normal liver. Immunostaining for TFF1 and hematoxylin. A, Only a few biliary epithelial cells (arrow) expressed TFF1 in the supranuclear area in the large bile ducts; $\times 40$. B, Biliary epithelial cells in the small bile ducts (arrow) do not express TFF1; $\times 40$.

ICC of peripheral type (Table 2). TFF1 expression was decreased in poorly differentiated ICC when compared with well-differentiated to moderately differentiated ICC.

\section{Expression of TFF1 mRNA}

In situ hybridization: Positive signals of TFF1 mRNA were detected in the cytoplasm of carcinoma cells and BEC (Fig. 4B); this corresponded to the expression of TFF1 protein, topologically. TFF1 mRNA expression was not detected by in situ hybridization in the invasive ICC in which TFF1 protein was not expressed (Fig. 4D). The signals were completely negative when a sense probe was applied instead of an antisense probe.

Semiquantitative RT-PCR: A high level of TFF1 mRNA expression was detected in the samples taken from noninvasive ICC, whereas it was lower or absent in the samples taken from invasive ICC with no expression of TFF1 protein despite having almost the same level of glyceraldehyde-3-phosphate dehydrogenase (GAPDH) expression (Fig. 5).

\section{Relationship of TFF1 and MUC5AC Expression}

To evaluate the relationships between TFF1 and MUC5AC expression, we selected 24 foci in the spec-
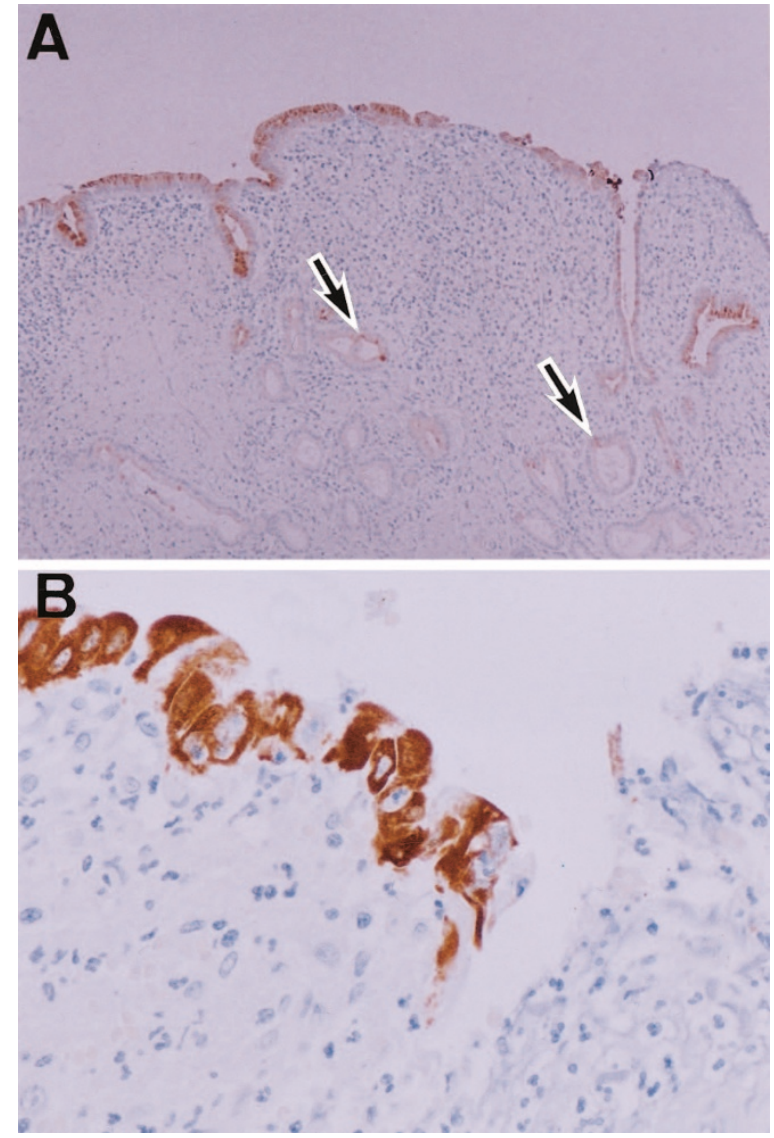

Figure 2.

TFF1 expression in hepatolithiasis. Immunostaining for TFF1 and hematoxylin. A, Most biliary epithelial cells are in the large bile duct, and focal peribiliary glands (arrows) express TFF1; $\times 20$. B, Biliary epithelial cells at the margin of erosion express TFF1 extensively; $\times 40$.

imens of 10 ICC associated with hepatolithiasis and 31 foci in the specimens of 10 ICC alone, including foci of invasive ICC, noninvasive ICC, biliary epithelial dysplasia, and BEC in the large bile ducts. In biliary epithelial dysplasia and noninvasive ICC, both TFF1

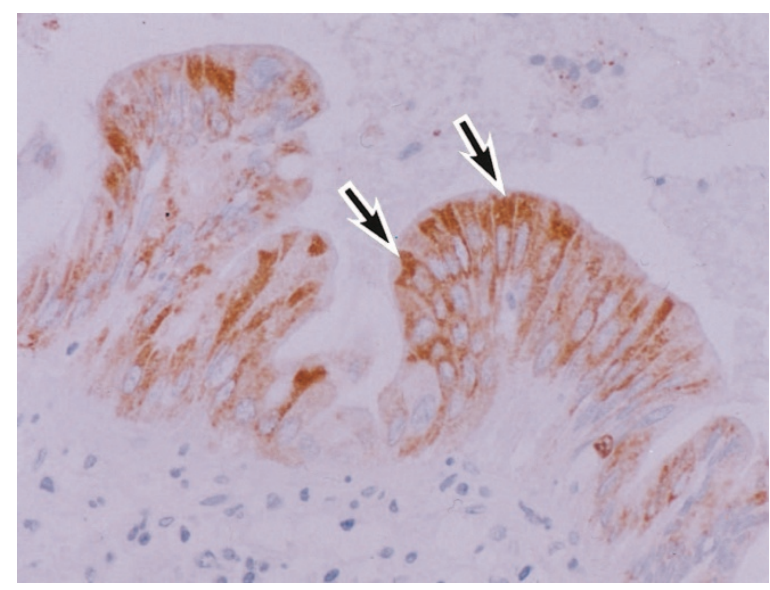

Figure 3.

TFF1 expression in dysplasia associated with hepatolithiasis. Biliary epithelial dysplasia expresses TFF1 extensively in the supranuclear region of each cell (arrows). Immunostaining for TFF1 and hematoxylin. 

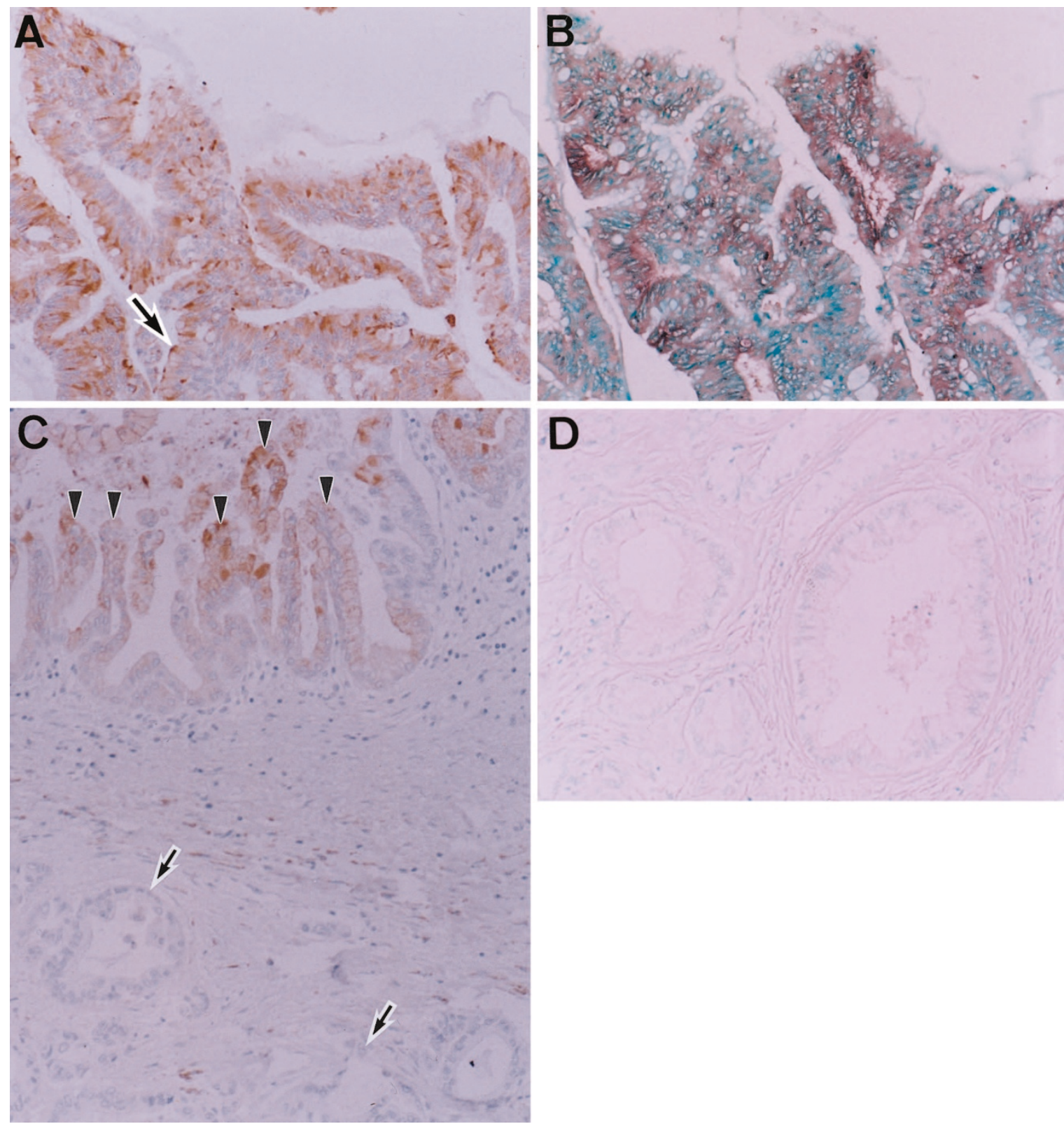

\section{Figure 4.}

TFF1 expression in noninvasive and invasive cholangiocarcinoma associated with hepatolithiasis. A, Carcinoma cells showing papillary growth in the large bile ducts express TFF1 protein extensively. Positive staining is seen in the supranuclear area and focally apical surface (arrows) extensively. Noninvasive cholangiocarcinoma. Immunostaining for TFF1 and hematoxylin; $\times 40$. B, TFF1 mRNA expression is observed in the same area as TFF1 protein. In situ hybridization and methyl green; $\times 40$. C, TFF1 expression in invasive cholangiocarcinoma associated with hepatolithiasis. Carcinoma cells of the surface noninvasive area express TFF1 (arrowheads), whereas the invasive area is negative for TFF1 (arrows). Immunostaining for TFF1 and hematoxylin; $\times 20$. D, TFF1 mRNA expression is not detected at the lesion of invasive cholangiocarcinoma; $\times 40$.

and MUC5AC were equally up-regulated, and TFF1 and MUC5AC were mainly colocalized histologically (Figs. 4C and 6). The expression of TFF1 was frequently decreased or lost at the foci of invasive ICC (Fig. 4C), whereas MUC5AC expression was usually preserved at the same foci (Fig. 6).

\section{Ki-67 Labeling Index (LI)}

The Ki-67 LI of TFF1-expressing ICC (LI $12.9 \pm 8.5 \%)$ tended to be slightly lower than that of TFF1-negative
ICC $(17.4 \pm 7.7 \%)$. However, there was no significant difference.

\section{Mutational Analysis of the TFF1 gene}

We detected a total of four somatic mutations of the TFF1 gene in two $(28.6 \%)$ of seven ICC with hepatolithiasis and one $(11 \%)$ of nine ICC, as summarized in Table 3. Of these four mutations, three were detected in noninvasive ICC with hepatolithiasis and one in invasive ICC of the peripheral type. A representative 
Table 2. Expression of TFF1 in ICC of Hilar Type and Peripheral Type

\begin{tabular}{|c|c|c|c|c|c|c|}
\hline & \multirow[b]{2}{*}{ (n) } & \multirow{2}{*}{$\begin{array}{c}\text { Positive rates } \\
(\%)\end{array}$} & \multicolumn{4}{|c|}{ Extent of expression } \\
\hline & & & 0 & $1+$ & $2+$ & $3+$ \\
\hline \multicolumn{7}{|l|}{ Invasive ICC alone } \\
\hline Hilar type & 10 & $70 \%$ & 3 & 4 & 3 & 0 \\
\hline Peripheral type & 8 & $62.5 \%$ & 3 & 3 & 1 & 1 \\
\hline
\end{tabular}

The rate of TFF1-expressing cells is $0,<5 \% ; 1+, 5-30 \% ; 2+, 30-70 \% ; 3+,>70 \%$.

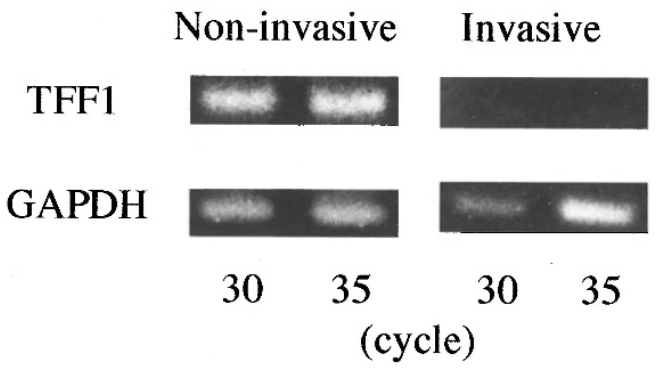

Figure 5.

Semiquantitative analysis of TFF1 mRNA in the noninvasive and invasive parts of a cholangiocarcinoma by RT-PCR. TFF1 mRNA expression was clearly detected in the noninvasive part, whereas its expression was absent in the invasive part despite having almost the same level of glyceraldehyde-3phosphate dehydrogenase as an internal control.

mutation is shown in Figure 7. All mutations were missense and situated at three codons (codons 12, 22 , and 33) in exon 2. Three were located in loop I and loop II structures of TFF1, and the remaining one was at the cysteine residue forming loop I (Fig. 7). All of the amino acid residues in which mutation was detected were at highly conserved residues between human and rodent species (Park et al, 2000). No mutation was detected in DNA samples from hepatolithiasis without dysplasia and carcinoma.

\section{LOH analysis of the TFF1 gene}

Of seven cases of ICC with hepatolithiasis, two (28.6\%) were homozygotes lacking the BsrD1 recognition site (T/T genotype). Five (71.4\%) were informative, showing heterozygotes ( $\mathrm{C} / \mathrm{T}$ genotype), and none had LOH in RFLP analysis. In nine cases of ICC alone, two $(22.2 \%)$ were homozygotes of the T/T genotype, seven $(77.8 \%)$ were informative, but none showed LOH (Table 3).

\section{Promotor Methylation Analysis of the TFF1 Gene}

Figure 8B shows a representative result from PCR of DNA after digestion with the methylation-sensitive Hpal and methylation-insensitive Mspl restriction enzymes. In five of six noninvasive ICC with TFF1 expression, DNA was sensitive for Hpall digestion (Fig. $8 B$ ). This suggested that neither of the restriction sites (Fig. 8A) were methylated. In contrast, DNA was resistant for Hpall digestion in invasive ICC in which TFF1 expression was lost (Fig. 8B). These results indicated that both restriction sites in the TFF1 promoter region were methylated. Figure $8 \mathrm{C}$ shows the

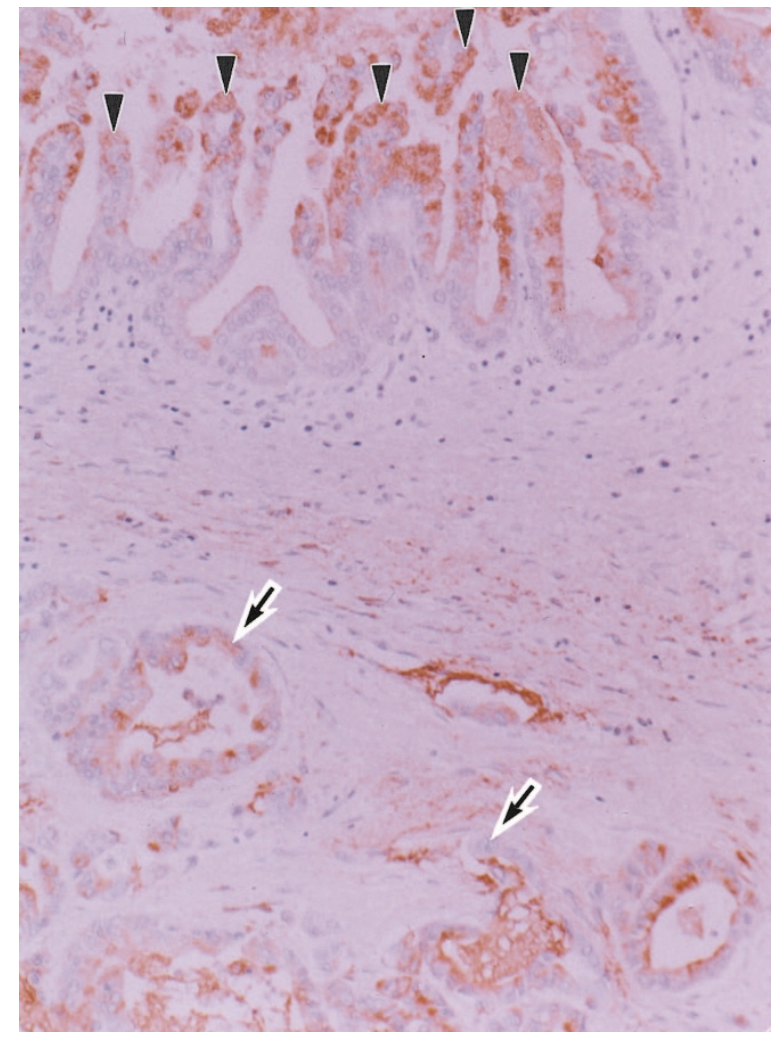

Figure 6.

MUC5AC expression in cholangiocarcinoma associated with hepatolithiasis. Both the carcinoma cells of the surface noninvasive area (arrowheads) and the invasive area (arrows) express MUC5AC. Immunostaining for MUC5AC and hematoxylin; $\times 20$.

correlation of the immunohistochemical expression of TFF1 gene products with the methylation status of the TFF1 promoter region. The expression of TFF1 gene products correlated well with methylation of the TFF1 promoter region (Fig. $8 \mathrm{C}$ ). These results point to the involvement of promoter methylation in the loss of TFF1 expression in invasive ICC.

\section{Discussion}

There is accumulating evidence that TFF1 has tumorsuppressor properties, particularly in the stomach (Calman et al, 1999; Gott et al, 1996; Henry et al, 1991; Lefebvre et al, 1996; Machado et al, 2000; Nishizuka et al, 1998; Park et al, 2000; Sakata et al, 1997; Taupin et al, 2001). In this study, we evaluated the participation of TFF1 in cholangiocarcinogenesis in hepatolithiasis. Although BEC in control livers expressed TFF1 
Table 3. Genetic Analysis and Immunoreactivity of TFF1 in ICC

\begin{tabular}{|c|c|c|c|c|c|}
\hline Sample no. & Age/sex & Histologic type & Mutations & $\mathrm{LOH}$ & Immunostaining \\
\hline \multicolumn{6}{|c|}{ ICC with hepatolithiasis $(n=7)$} \\
\hline 3 & $64 / \mathrm{M}$ & Papillary, noninvasive & GTC to $\underline{A T C}(\mathrm{~V} 22$ I) & - & + \\
\hline 5 & $70 / F$ & Papillary, noninvasive & $\begin{array}{l}\text { CGT to TGT (R12 C) } \\
\text { GTC to GAC (V22 D) }\end{array}$ & - & + \\
\hline \multicolumn{6}{|l|}{ ICC alone $(n=9)$} \\
\hline 5 & $65 / F$ & Tubular, poorly diff., invasive & 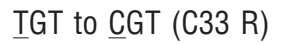 & - & - \\
\hline
\end{tabular}

poorly diff., poorly differentiated; LOH, loss of heterozygosity.

A

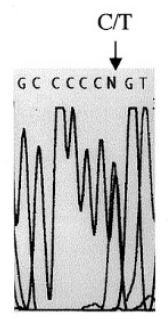

$\mathrm{C}$

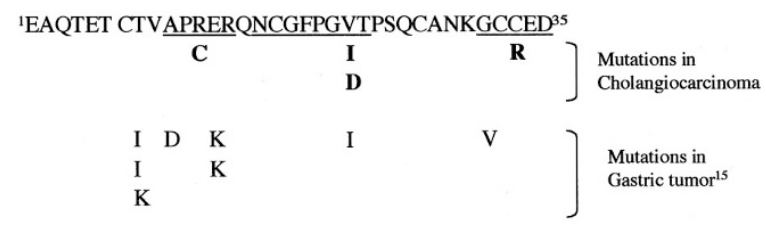

Figure 7.

TFF1 gene mutations in cholangiocarcinoma. A, Missense mutation at codon 12 (CGT to TGT) in exon 2 of TFF1. B, Missense mutation at codon 22 (GTC to GAC) in exon 2 of TFF1. C, Amino acid sequence of human TFF1 peptides. The underlined sequences are identical to TFF1 of rat and mouse.

at a low level, aberrant expression of TFF1 was mildly found in BEC in all cases of hepatolithiasis. Furthermore, TFF1 was moderately to extensively expressed in the biliary epithelial dysplasia and noninvasive ICC, indicating that TFF1 is up-regulated in the intrahepatic biliary tree during the stepwise carcinogenesis in hepatolithiasis. TFF1 mRNA expression detected by in situ hybridization, and semiquantitative RT-PCR was consistent with TFF1 protein overexpression.

How does up-regulation of TFF1 occur during cholangiocarcinogenesis in hepatolithiasis? Three TFF genes coded on chromosome 21q22.3 and the MUC2, MUC5AC, and MUC6 mucin genes coded on 11p15.5 have a characteristic expression pattern along the gastrointestinal tract (Chang et al, 1994; Lefebvre et al, 1993; Suemori et al, 1991; Tytgat et al, 1995). The colocalization pattern of TFF1 and MUC5AC, which is regarded as a gastric surface/foveolar phenotype (Machado et al, 2000), is also observed in the ulcerassociated cell lineage in Crohn's disease (Longman et al, 2000). In this study, we demonstrated that MUC5AC was closely associated with TFF1 in BEC in biliary epithelial dysplasia and noninvasive ICC with hepatolithiasis. It seems plausible, therefore, that TFF1 and MUC5AC might have been overexpressed simultaneously in BECs undergoing gastric metaplasia in hepatolithiasis (Sasaki et al, 1996). The intrahepatic biliary tree in hepatolithiasis is known to frequently
A $\quad-218$

-218 2 CTCAGATCCCTCAGCCAAGA TAGCCTCACCACATGTCGTCTCTGTCTATC

AGCAAATCCTTCCATGTAGCTTGACCATGTCTAGGAAACACCTTTGATA

AAAATCAGTGGAGATTATTGTCTCAGAGGATCCC $C^{*} G$ GGCCTCCTTAGg

CAAATGTTATCTAA CG CTCTTTAAGCAAACAGAGCCTACCCTATAAAAT

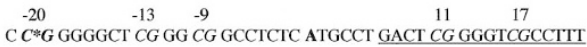

B

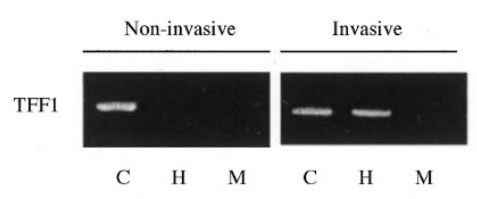

C

\begin{tabular}{|c|c|c|c|c|}
\hline & Non-Invasive & & Invasive & \\
\hline & TFF1-IH & Нра II & TFF1-IH & Hра II \\
\hline \multicolumn{5}{|l|}{ ICC case 1} \\
\hline case 2 & & & $\square$ & 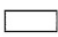 \\
\hline case 3 & & & $\square$ & 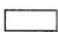 \\
\hline case 4 & & & $\square$ & 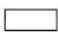 \\
\hline case 5 & & & NA & NA \\
\hline case 6 & & & NA & NA \\
\hline case 7 & NA & NA & 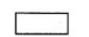 & 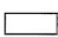 \\
\hline case 8 & NA & NA & 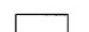 & 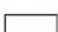 \\
\hline
\end{tabular}

Figure 8.

A, Partial promoter sequence of TFF1. Asterisk indicates restriction sites ( Hpall or Mspl). Underlined bases indicate sequences used to design primers for the PCR assay for methylation of CpG sites. Bold $A$ indicates the postulated start of the TFF1 polypeptide. Italic indicates CpG sites at $-84,-56,-20,-13$, $-9,11$, and 17. B, PCR of DNA isolated from microdissected tissue after digestion by restriction enzymes. DNA isolated from tissues was digested by Mspl $(M)$ or the methylation sensitive Hpall $(H)$ as described in "Materials and Methods." PCR was performed using the primers in $A$ and Described in "Materials and Methods." $C=$ control (not digested DNA). The DNA sample from noninvasive intrahepatic cholangiocarcinoma (ICC) was sensitive to both Hpall and Mspl, whereas the DNA sample from invasive ICC was highly resistant to Hpall and sensitive to Mspl. C, Correlation of the immunohistochemical expression of TFF1 gene products with promoter methylation in noninvasive and invasive ICC. Filled symbols indicate positive tissue immunostaining or Hpall sensitivity (unmethylated). Open symbols indicate negative reactions. $N A=$ not available

present gastrointestinal metaplasia potentially followed by the development of biliary epithelial dysplasia and then ICC (Sasaki et al, 1996, 1998). In the metaplastic and early neoplastic lesions of the gastrointestinal tract, the coordinated expression of TFF1/ 
MUC5AC remains, implying the preservation of coordinated regulation between chromosome 21q22.3 and 11 15.5 regions and a synergistic role of TFF1/ MUC5AC to protect and reconstitute epithelial tissue. This regulation by MUC5AC/TFF1 may be maintained in the biliary epithelial dysplasia and noninvasive ICC in the intrahepatic biliary tree in hepatolithiasis.

Interestingly, TFF1 expression was significantly decreased in invasive ICC with hepatolithiasis, suggesting that loss of TFF1 expression may be related to the invasive growth of ICC. By semiquantitative RT-PCR and in situ hybridization, the changes in TFF1 expression were shown to be a result of the differences in transcriptional processing, not translational processing. This study disclosed that although the expression of MUC5AC gastric mucin was still well preserved in invasive ICC, the expression of TFF1 was clearly decreased in invasive ICC.

This indicates that the decreased expression of TFF1 does not simply reflect the loss of gastric phenotype and that the coordinated regulation of TFF1/ MUC5AC expression is disrupted in invasive ICC. Furthermore, the decreased TFF1 expression seems not to reflect simply the loss of differentiation, because decreased TFF1 expression was also seen in well-differentiated invasive ICCs in this study. The loss of TFF1 expression is detected in $44.2 \%$ of gastric carcinoma, although the difference in TFF1 expression between noninvasive and invasive gastric cancer is not clear (Park et al, 2000). Because TFF1 is a candidate tumor suppressor gene and because TFF1 downregulates cell proliferation and prevents tumor cell growth in in vitro study (Calman et al, 1999), decreased TFF1 might lead to the acceleration of tumor cell growth and the development of invasive and advanced ICC in the same way as gastric cancer. In fact, the Ki-67 LI of TFF1-expressing ICC tended to be slightly lower than that of TFF1-negative ICC in the present study. Taken together, the decreased expression of TFF1 in invasive ICC may support the role of TFF1 inactivation in cholangiocarcinogenesis as well as gastric carcinogenesis, in agreement with the results obtained in TFF1 knockout mice (Lefebvre et al, 1996).

The expression of TFF1 in ICC alone was similar in frequency and degree to that seen in invasive ICC with hepatolithiasis. There are several possible explanations for this phenomenon. First, TFF1 might have been expressed in the diseased bile ducts from which ICC might have arisen, as discussed in the scenario of ICC development and progression in hepatolithiasis. In fact, Srivatsa et al (2002) recently reported that several types of diseased bile ducts express TFF1 and TFF3 frequently. Second, the expression of TFF1 in ICC cells may have another pathologic significance. For example, it is known that TFF may be up-regulated by transforming growth factor- $\alpha$ (TGF- $\alpha$ ) as a coordinate function of TGF- $\alpha$ /epidermal growth factor receptor (EGFR) (Beauchamp et al, 1989; Cook et al, 1997; Taupin et al, 1999). There is recent evidence that TFF3 treatment of cell lines causes EGFR phosphorylation, and EGFR activation is necessary for TFF3- mediated up-regulation of TFF1 and TFF2 (Nunez et al, 1989; Taupin et al, 1999). An EGF receptor ligand, TGF- $\alpha$, which is an epithelial mitogen (Beauchamp et al, 1989) may up-regulate TFF1 expression simultaneously with activation of cell proliferation. Thus, TFF1 expression may be involved in the disordered cell kinetics of carcinoma cells in invasive ICC.

Mutational analysis revealed four missense mutations of the TFF1 gene in ICC with and without hepatolithiasis. On the other hand, no mutation was detected in any cases of hepatolithiasis without dysplasia and carcinoma. All of the mutations detected in ICC were confined to the loop I and loop II structure of TFF1 as reported in gastric cancer (Park et al, 2000) and in highly conserved residues between human and rodent species. It is of interest that two mutations are targeted to codon 22 (V22 I in Case 3 and V22 D in Case 5). The same mutation (V22 I) was already detected in gastric adenoma (Park et al, 2000). The mutation at codon 22 may lead to the impairment of biologic activities of TFF1. The other two mutations detected in this study have not been reported so far. A mutation at codon $12(\mathrm{R} 12 \mathrm{C})$ provides a new cysteine residue that may alter the combination of disulfide bonds and the structure of the TFF1 molecule. Another mutation at codon 33 (C33 R) seems to change the structure of TFF1, because the cysteine that constitutes loop I is lost. It is likely that this altered structure causes dysfunction of TFF1. More studies are necessary to clarify the exact roles of TFF1 mutation in carcinogenesis and tumor progression of ICC.

In the present study, there was no correlation between immunoreactivity and genetic alterations of the TFF1 gene as reported in gastric carcinoma (Park et al, 2000). Because the mutations were detected in both noninvasive and invasive parts of ICC, the mutations themselves could not explain the decreased TFF1 expression at the invasive site. The decreased TFF1 expression in the invasive area of ICC could not be explained by $\mathrm{LOH}$. To address this issue, we analyzed the methylation status of the TFF1 promoter region and examined the correlation with TFF1 expression in noninvasive and invasive ICC. Recent reports suggest that methylation of the TFF1 promoter region may underlie the loss of TFF1 in gastric carcinoma (Carvalho et al, 2002; Fujimoto et al, 2000). As a result, the expression of TFF1 gene products correlated well with methylation of the TFF1 promoter region. That is, the TFF1 promoter region was unmethylated in noninvasive ICC with TFF1 expression, whereas the TFF1 promoter was methylated in invasive ICC in which TFF1 expression was lost. These findings clearly indicate that the loss of TFF1 expression in invasive ICC could be explained by promoter methylation. The hypermethylation of the TFF1 promoter occurs as an event in the cholangiocarcinogenesis pathway. Unusually, methylation of the TFF1 promoter was detected in one of six noninvasive ICC despite immunohistochemical TFF1 expression. A subclone of carcinoma cells that did not express TFF1 promoter methylation in noninvasive ICC may have caused the positive signal in this case. Similar epigenetic changes 
caused by hypermethylation of the promoter region in carcinoma have been reported in tumor suppressor genes such as p16 and p14 (Herman et al, 1996; Lee et al, 2002) and were related to carcinogenesis and tumor progression.

On the other hand, abundantly secreted TFF1 coupled with MUC5AC in hepatolithiasis may contribute to raising the viscosity of biliary mucin followed by lithogenesis. TFF peptides have also been proposed to act as link peptides and thereby to influence the rheologic properties of mucous gels (Hauser et al, 1993). TFFs alter the physiologic properties of the secreted mucin, leading to an increase in the optical density and viscosity of purified mucin preparations when added in vitro (Babyatsky et al, 1996). TFF1interacting proteins are MUC2 and MUC 5AC, and the binding regions are VWFC1 and VWFC2 cysteine-rich domains, possibly raising the viscosity of mucin (Tomasetto et al, 2000). It is well known that oversecreted mucin from proliferated peribiliary glands in hepatolithiasis is essential for forming stones because mucin constructs the lamellar backbone of calciumbilirubinate stones (Nakanuma et al, 1988, 1994; Nakayama et al, 1986). Our previous report (Sasaki et al, 1998) revealed an increased expression of MUC5AC apomucin, which is a major gastric apomucin having the ability of gel formation, suggesting its critical role in lithogenesis. Therefore, increased TFF1 seems to bind to MUC5AC, contributing to increase the viscosity of biliary mucin secreted in the intrahepatic bile ducts and then formation of hepatoliths.

In conclusion, up-regulation of TFF1 coupled with MUC5AC in BEC in hepatolithiasis and at early stages of cholangiocarcinogenesis may reflect gastric metaplasia of the intrahepatic biliary tree in hepatolithiasis. In the situation of the intrahepatic biliary tree with gastric metaplasia, a decrease or loss of TFF1 expression may lead to increased cell proliferation and then to the invasive character of ICC as speculated in gastric cancer. Promoter methylation of TFF1 was well correlated to the decreased expression of TFF1. Somatic mutations of the TFF1 gene detectable in ICC were at least partly responsible for the dysregulation of TFF1 in ICC, although $\mathrm{LOH}$ was not detectable in the TFF1 gene. TFF1 expression in BEC with MUC5AC expression in the intrahepatic large bile duct may be involved in stone formation in hepatolithiasis.

\section{Materials and Methods}

\section{Classification of Intrahepatic Biliary Tree and Definition of Biliary Epithelial Dysplasia}

The intrahepatic biliary tree was divided into the intrahepatic large bile ducts and small bile ducts as previously described (Nakanuma and Sasaki, 1989; Terada and Nakanuma, 1993; Terada et al, 1987). Biliary epithelial dysplasia identified in the intrahepatic large bile ducts was defined as previously described (Terada and Nakanuma, 1992).

\section{Patients and Liver Tissue Processing}

A total of 111 liver tissue specimens were collected from the surgical and autopsy files of our laboratory and affiliated hospitals. The specimens included 16 ICC with hepatolithiasis (10 invasive ICC, 6 noninvasive ICC), 11 biliary epithelial dysplasia with hepatolithiasis, 16 hepatolithiasis alone, and 18 usual invasive ICC without hepatolithiasis. As controls, 27 histologically normal livers and 12 livers with EBO were examined. Eleven cases of hepatocellular carcinoma were also examined. Hepatoliths were all of calcium bilirubinate stones. Ten invasive ICC cases with hepatolithiasis were composed of six well-differentiated to moderately differentiated ICC and four poorly differentiated ICC. All of six noninvasive ICC with hepatolithiasis were well-differentiated to moderately differentiated carcinomas. Eighteen ICC alone were subdivided into 10 of the hilar type and 8 of the peripheral type according to Okuda et al (1977); they were composed of 14 well-differentiated to moderately differentiated ICC and 4 poorly differentiated ICC.

Several tissue samples, including the intrahepatic large bile ducts, were obtained from individual livers, fixed in 10\% neutral buffered formalin, and embedded in paraffin. Twenty sections, 4- $\mu \mathrm{m}$ thick, were cut from each paraffin block. One was stained with hematoxylin and eosin, and the rest were processed for the following studies.

\section{Immunohistochemistry and Semiquantitative Evaluation}

Immunodetection of TFF1 and MUC5AC. TFF1 protein and MUC5AC gastric type mucin were detected by immunohistochemical staining using mouse monoclonal anti-pS2 protein antibody (clone BC04, dilution 1:200; Dako, Santa Barbara, California) and mouse mAb 45M1 (dilution 1:100; Novocastra, Newcastle, United Kingdom), and the Envision + system (mouse/ horseradish peroxidase; Dako). The extent of TFF1 and MUC5AC expression was semiquantitatively scored in each specimen: score 0 , the percentage of positive cells was $<5 \%$, if present; score $1,5 \%$ to $30 \%$; score 2, 31\% to $70 \%$; score 3, $>70 \%$.

$\mathrm{LI}$ of Ki-67 in TFF1-Positive and -Negative ICC. Proliferative activities of TFF1-positive and -negative ICC cells were examined immunohistochemically using mouse mAb against Ki-67 antigen (MIB-1, dilution 1:50; Dako). A Ki-67 LI for the evaluation of cellproliferative activity was calculated by counting positive cells among 1000 nuclei. The Ki-67 LI (\%) in TFF1 expressing ICC and that of TFF1-negative ICC were assessed in three cases of ICC with hepatolithiasis and two cases of ICC alone using serial sections immunostained for TFF1 and Ki-67, respectively.

\section{Detection of TFF1 mRNA by In Situ Hybridization and Semiquantitative RT-PCR}

Sections from 10 cases of hepatolithiasis with ICC, 5 without ICC, and 3 EBO were used for in situ hybridization. Sections from seven ICC associated with 
hepatolithiasis and nine invasive ICC alone were used for semiquantitative RT-PCR of TFF1 mRNA.

\section{In Situ Hybridization}

In situ hybridization was performed as previously described (Sasaki et al, 1998). DIG-labeled RNA probes for TFF1 were derived from RT-PCR products (Cone and Schlaepfer, 1997) using the following primers: TFF1 forward, 5'-TTTGGAGCA-GAGAGGAGG; reverse, 5'-TTGAGTAGTCAA AGTCAGAGCAG (PCR products, $438 \mathrm{bp}$ ) (Wiede et al, 1999).

\section{Semiquantitative RT-PCR of TFF1 mRNA}

A total RNA was isolated from the targeted noninvasive and invasive ICC tissue using a Pinpoint slide RNA isolation system II (Zymo Research, Orange, CA). After cDNA was synthesized, semi-quantitative RTPCR using the appropriate number of cycles for linear amplification and comparison with GAPDH as an internal control was carried out. The intron-spanning PCR primer sets for the amplification of the TFF1 and GAPDH were as follows; TFF1 forward, 5'-CCATGGAGAACAAGGTGATCTG; reverse, 5'-ACCACAATTCTGTCTTTCACGG (PCR products, 119bp); GAPDH forward, 5'-CGACAGTCAGCCGCATCTT; reverse, 5'TTCCCCATG-GTGTCTGAGC (PCR products, 65bp).

\section{Mutational and LOH Analysis}

Formalin-fixed, paraffin-embedded sections from seven ICC (one noninvasive ICC and six invasive ICC with foci of noninvasive ICC) associated with hepatolithiasis and nine invasive ICC alone were used for the mutational and LOH analysis of the TFF1 gene. Four cases of hepatolithiasis without dysplasia and carcinoma were also used for mutational analysis.

Tissue Microdissection and DNA Extraction. ICC tissues and nontumorous tissues were scraped using a surgical blade from the remaining sections. The DNA were extracted using DEX-PAT solution (Takara, Tokyo).

PCR. Standard PCR was performed. The PCR primer sets for the amplification of the TFF1 exons 1, 2, and 3 were synthesized (Hokkaido System Science, Sapporo) according to the method of Park et al (2000).

Direct Sequence. The direct sequencing of PCR products for TFF1 exon 2 was performed using the Big Dye cyclic sequencing kit (Perkin-Elmer, Foster City, California) and the $\mathrm{ABI} 310$ sequencer (Perkin-Elmer). The mutation sites were detected by comparison with the sequence obtained from GenBank (Accession No. $\mathrm{X} 05321)$.

PCR-Single-Strand Conformation Polymorphism. The PCR products were denatured for 5 minutes at $95^{\circ} \mathrm{C}$ in a $1: 1$ dilution of sample buffer containing $98 \%$ formamide and $5 \mathrm{mmol} / \mathrm{L} \mathrm{NaOH}$ and were loaded onto a single-strand conformation polymorphism gel (Amersham Pharmacia Biotech, Amersham Place, United Kingdom) and subjected to electrophoresis at $15 \mathrm{~W}$, $10^{\circ} \mathrm{C}$ for 100 minutes. After electrophoresis, the gels were stained using a silver staining kit (Amersham
Pharmacia Biotech). DNA showing mobility shifts were cut out from the gel and reamplified using the same primer set. Sequencing of the PCR products was performed as described above.

$\mathrm{LOH}$ Analysis. An intragenic polymorphic marker for $\mathrm{LOH}$ analysis of the TFF1 gene was examined in seven cases of ICC with hepatolithiasis and in nine cases of ICC alone by RFLP analysis according to the method of Park et al (2000).

\section{Promoter Methylation Analysis of the TFF1 Gene}

Genomic DNA was isolated as described above from each of six microdissected foci of noninvasive and invasive ICC. DNA was digested with either Hpall (methylation sensitive) or Mspl (methylation insensitive) restriction enzymes (New England BioLabs, Beverly, Massachusetts) for 24 hours at $37^{\circ} \mathrm{C}$. The digested or control (nondigested) DNA samples were amplified by semi-nested PCR using the following primers (Fig. 8A): 5'CTCAGATCCCTCAGC-CAAGA (forward for the first PCR); 5'GCCAAGATGACCTCACCACA (forward for semi-nested PCR); and 5'AAAGGCGACCCCGAGTC (reverse) (final PCR product: $227 \mathrm{bp}$ ). Each amplification was performed for a total of 40 cycles.

\section{Statistical Analysis}

The difference among groups was assessed by the Wilcoxon rank sum test. A $p$ value less than 0.01 was regarded as significant.

\section{References}

Babyatsky M, DeBeaumont M, Thim L, and Podolsky D (1996). Oral trefoil peptides protect against ethanol- and indomethacin-induced gastric injury in rats. Gastroenterology 110:489-497.

Beauchamp R, Bernard J, McCutchen C, Cherner C, and Coffey R (1989). Localization of transforming growth factor alpha and its receptor in gastric mucosal cells: Implications for a regulatory role in acid secretion and mucosal renewal. $\mathrm{J}$ Clin Invest 84:1017-1023.

Beck S, Schmitt H, Shizuya H, Blin N, and Gott P (1996). Cloning of continuous genomic fragments from human chromosome 21 harboring three trefoil peptide genes. Hum Genet 98:233-235.

Calman D, Westley B, May F, Floyd D, Marchbank T, and Playford R (1999). The trefoil peptide TFF1 inhibits the growth of the human gastric adenocarcinoma cell line AGS. J Pathol 188:312-317.

Carvalho R, Kayademir T, Soares P, Canedo P, Sousa S, Oliveira C, Leistenschneider P, Seruca R, Gott P, Blin N, Carneiro F, and Machado JC (2002). Loss of heterozygosity and promoter methylation, but not mutation, may underlie loss of TFF1 in gastric carcinoma. Lab Invest 82:1319-1326.

Chang SK, Dohrman AF, Basbaum CB, Ho SB, Tsuda T, Toribara NW, Gum JR, and Kim YS (1994). Localization of mucin (MUC2 and MUC3) messenger RNA and peptide expression in human normal intestine and colon cancer. Gastroenterology 107:28-36. 
Cone R and Schlaepfer E (1997). Improved in situ hybridization of HIV with RNA probes derived from PCR products. J Histochem Cytochem 45:721-727.

Cook GA, Yeomans ND, and Giraud AS (1997). Temporal expression of trefoil peptides in the TGF-alpha knockout mouse after gastric ulceration. Am J Physiol 272:G1540G1549.

Fujimoto J, Yasui W, Tahara H, Tahara E, Kudo Y, and Yokozaki H (2000). DNA hypermethylation at the pS2 promoter region is associated with early stage of stomach carcinogenesis. Cancer Lett 149:125-134.

Gott P, Beck S, Machado J, Camiero F, Schmitt H, and Blin $N$ (1996). Human trefoil peptides: Genomic structure in $21 \mathrm{q} 22.3$ and coordinated expression. Eur J Hum Genet 4:308-315.

Hauser F, Poulsom R, Chinery R, Rogers L, Hanby A, Wright N, and Hoffmann W (1993) hP1.B, a human P-domain peptide homologous with rat intestinal trefoil factor, is expressed also in the ulcer-associated cell lineage and the uterus. Proc Natl Acad Sci USA 90:6961-6965.

Henry J, Bennett M, Piggott N, Levett D, May F, and Westley $B$ (1991). Expression of the pNR-2/pS2 protein in diverse human epithelial tumors. Br J Cancer 64:677-682.

Herman JG, Graff JR, Myohanen S, Nelkin BD, and Baylin SB (1996). Methylation-specific PCR: A novel PCR assay for methylation status of $\mathrm{CpG}$ islands. Proc Natl Acad Sci USA 93:9821-9826.

Lee S, Kim WH, Jung HY, Yang MH, and Kang GH (2002). Aberrant $\mathrm{CpG}$ island methylation of multiple genes in intrahepatic cholangiocarcinoma. Am J Pathol 161:1015-1022.

Lefebvre O, Chenard M, Masson R, Linares J, Dierich A, Lemeur M, Wendling C, Tomasetto C, Chambon P, and Rio $M$ (1996). Gastric mucosa abnormalities and tumorigenesis in mice lacking the pS2 trefoil protein. Science 274:259-262.

Lefebvre O, Wolf C, Kedinger M, Chenard MP, Tomasetto C, Chambon P, and Rio MC (1993). The mouse one P-domain (pS2) and two P-domain (mSP) genes exhibit distinct patterns of expression. J Cell Biol 122:191-198.

Longman RJ, Douthwaite J, Sylvester PA, Poulsom R, Corfield AP, Thomas MG, and Wright NA (2000). Coordinated localisation of mucins and trefoil peptides in the ulcer associated cell lineage and the gastrointestinal mucosa. Gut 47:792-800.

Machado J, Nogueira A, Carmeiro F, Reis C, and SobrinhoSimoes M (2000). Gastric carcinoma exhibits distinct types of cell differentiation: An immunohistochemical study of trefoil peptides (TFF1 and TFF2) and mucins (MUC1, MUC2, MUC5AC, and MUC6). J Pathol 190:437-443.

Nakanuma $Y$ and Sasaki M (1989). Expression of bloodgroup-related antigens in the intrahepatic biliary tree and hepatocytes in normal livers and various hepatobiliary diseases. Hepatology 10:174-178.

Nakanuma Y, Sasaki M, Terada T, and Harada K (1994). Intrahepatic peribiliary glands of humans. II. Pathological spectrum. J Gastroenterol Hepatol 9:80-86.

Nakanuma Y, Terada T, Tanaka Y, and Ohta G (1985). Are hepatolithiasis and cholangiocarcinoma aetiologically related? A morphological study of 12 cases of hepatolithiasis. Virchows Arch A 406:45-58.
Nakanuma Y, Yamaguchi K, Ohta G, and Terada T (1988). Pathologic features of hepatolithiasis in Japan. Hum Pathol 19:1181-1186.

Nakayama F, Soloway R, and Nakama T (1986) Hepatolithiasis in East Asia: Retrospective study. Dig Dis Sci 31:21-26.

Nishizuka S, Tamura G, Terashima M, and Satodate R (1998). Loss of heterozygosity during the development and progression of differentiated adenocarcinoma of the stomach. J Pathol 185:38-43.

Nunez A, Berry M, Imler J, and Chambon P (1989). The $5^{\prime}$ flanking region of the $\mathrm{pS} 2$ gene contains a complex enhancer region responsive to oestrogens, epidermal growth factor, a tumour promotor (TPA), the c-Ha-ras oncoprotein and the c-jun protein. EMBO J 8:823-829.

Okuda K, Kubo Y, Okazaki N, Arishima T, and Hashimoto M (1977). Clinical aspects of intrahepatic bile duct carcinoma including hilar carcinoma: A study of 57 autopsy-proven cases. Cancer 39:232-246.

Park S, Oh R, Park J, Lee J, Shin M, Kim H, Lee H, Kim Y, Kim S, Lee S, Yoo N, and Lee J (2000). Somatic mutations of the trefoil factor family gene in gastric cancer. Gastroenterology 119:691-698.

Podolsky D (1999). Mucosal immunity and inflammation. V. Innate mechanisms of mucosal defense and repair: The best offense is a good defense. Am J Physiol 277:G495-G499.

Poulsom R, Begos D, and Modlin I (1996). Molecular aspects of restitution: Functions of trefoil peptides. Yale $\mathrm{J}$ Biol Med 69:137-146.

Poulsom R and Wright N (1993). Trefoil peptides: A newly recognized family of epithelial mucin-associated molecules. Am J Physiol 265:9205-9213.

Rio M-C, Chenard M, Wolf C, Marcellin L, Tomasetto C, Lathe R, Bellocq J, and Chambon P (1991). Induction of pS2 and hSP genes as markers of mucosal ulceration of the digestive tract. Gastroenterology 100:375-379.

Sakata K, Tamura G, Nishizuka S, Maesawa C, Suzuki Y, Iwaya T, Terashima M, Saito K, and Satodate R (1997). Commonly deleted regions on the long arm of chromosome 21 in differentiated adenocarcinoma of the stomach. Genes Chromosome Cancer 18:318-321.

Sasaki M, Nakanuma Y, and Kim Y (1996). Characterization of apomucin expression in intrahepatic cholangiocarcinomas and their precursor lesions: An immunohistochemical study. Hepatology 24:1074-1078.

Sasaki M, Nakanuma Y, and Kim Y (1998). Expression of apomucins in the intrahepatic biliary tree in hepatolithiasis differs from that in normal liver and extrahepatic biliary obstruction. Hepatology 27:46-53.

Seib T, Blin N, Hilgert K, Seifert M, Theisinger B, Engel M, Dooley S, Zang K-D, and Welter C (1997). The three human trefoil genes TFF1, TFF2, and TFF3 are located within a region of $55 \mathrm{~kb}$ on chromosome 21q22.3. Genomics 40:200202.

Seitz G, Thelsinger B, Tomasetto G, Rio M-C, Chambon P, Blin N, and Welter G (1991). Breast cancer-associated protein pS2 expression in tumors of the biliary tract. Am J Gastroenterol 86:1491-1494. 
Srivatsa G, Giraud AS, Ulaganathan M, Yeomans ND, Dow C, and Nicoll AJ (2002). Biliary epithelial trefoil peptide expression is increased in biliary diseases. Histopathology 40:261268.

Suemori S, Lynch-Devaney K, and Podolsky DK (1991). Identification and characterization of rat intestinal trefoil factor: Tissue- and cell-specific member of the trefoil protein family. Proc Natl Acad Sci USA 88:11017-11021.

Taupin D, Pedersen J, Familari M, Cook G, Yeomans N, and Giraud AS (2001). Augmented intestinal trefoil factor (TFF3) and loss of pS2 (TFF1) expression precedes metaplastic differentiation of gastric epithelium. Lab Invest 81:397-408.

Taupin D, Wu D, Jeon W, Devaney K, Wang T, and Podolsky D (1999). The trefoil gene family are coordinately expressed immediate-early genes: EGF receptor- and MAP kinasedependent interregulation. J Clin Invest 103:R31-R38.

Terada T and Nakanuma Y (1992). Cell kinetics analyses and expression of carcinoembryonic antigen, carbohydrate antigen 19-9 and DU-Pan-2 in hyperplastic, preneoplastic and neoplastic lesions of intrahepatic bile ducts in hepatolithiasis. Virchow Arch A 420:327-335.

Terada T and Nakanuma $Y$ (1993). Development of human intrahepatic peribiliary glands: Histologic, keratin immunohistochemical, and mucus histochemical analyses. Lab Invest 168:261-269.

Terada T, Nakanuma Y, and Ohta G (1987). Glandular elements around the intrahepatic bile ducts in man: Their morphology and distribution in normal livers. Liver 7:1-8.
Thim $L$ (1989). A new family of growth factor-like peptides. FEBS Lett 250:85-90.

Tomasetto C, Masson R, Linares J, Wendling C, Lefebvre O, Chenard M, and Rio M (2000). pS2/TFF1 interacts directly with the VWFC cysteine-rich domains of mucins. Gastroenterology 118:70-80.

Tytgat KM, Bovelander FJ, Opdam FJ, Einerhand AW, Buller HA, and Dekker J (1995). Biosynthesis of rat MUC2 in colon and its analogy with human MUC2. Biochem J 309:221-229.

Wiede A, Jagla W, Welte T, Kohnlein T, Busk H, and Hoffmann W (1999). Localization of TFF3, a new mucusassociated peptide of the human respiratory tract. Am J Respir Crit Care Med 159:1330-1335.

Wright N, Hoffmann W, Otto W, Rio M-C, and Thim L (1997). Rolling in the clover: The trefoil factor family (TFF)-domain peptides, cell migration and cancers. FEBS Lett 408:121123.

Wright N, Poulsom R, Van Noorden S, Sarraf C, Elia G, Ahnen $\mathrm{D}$, Jeffery R, Longcroft J, Pike C, Rio M-C, and Chambon P (1993). Trefoil peptide gene expression in gastrointestinal epithelial cells in inflammatory bowel disease. Gastroenterology 104:12-20. 